\title{
More doctors providing abortion after federal rules change
}

\author{
n Cite as: CMAJ 2018 February 5;190:E147-8. doi: 10.1503/cmaj.109-5552
}

Posted on cmajnews.com on Jan. 16, 2018.

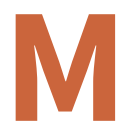

ore health professionals are providing the abortion drug Mifegymiso (mifepristone and misoprostol) since Health Canada eased prescribing restrictions in November. But gaps in coverage, and a requirement that women undergo an ultrasound to access the drug, remain barriers.

On Nov. 7, Health Canada opened the door for a wider range of health professionals to prescribe and dispense Mifegymiso; previously, only doctors were allowed to do so. "This has a massive impact on access, particularly for vulnerable populations," says Dr. Wendy Norman, who is studying the implementation of Mifegymiso in Canada.

Previously, "many doctors were not willing to be involved if they had to be stocking, managing and dispensing a drug," she explains. Although some provincial licensing bodies advised nurse practitioners and pharmacists to prescribe and dispense the drug anyway, many didn't want to go against federal rules.

Now that regulations have changed, "we've had about 30\% more people work with the collaboration of Canadian medical abortion providers," Norman says. "We have probably five times as many nurses as we had before, and a lot more rural family doctors."

Work is also underway to involve midwives in providing medical abortions, she says. "We've been working with them for almost a year now, and recently received a grant from the Canadian Institutes of Health Research to call a national meeting." Many other countries, including the United States, already allow midwives to prescribe and dispense Mifegymiso.

Among other changes, Health Canada no longer requires prescribers to com- plete special training, register with the drug's distributor or obtain written consent from patients. Women also have nine weeks to access Mifegymiso, up from a previous limit of seven.

However, a requirement that women undergo an ultrasound to rule out ectopic pregnancy before receiving the drug still stands. "It's based on evidence brought motion for Action Canada for Sexual Health and Rights. In Chilliwack, British Columbia, for example, "because there are just no ultrasound services in the city, you still end up having to travel for something that's not necessary."

Even in cities like Moncton, New Brunswick, "the province doesn't cover the cost of ultrasounds or abortions outside of hos-

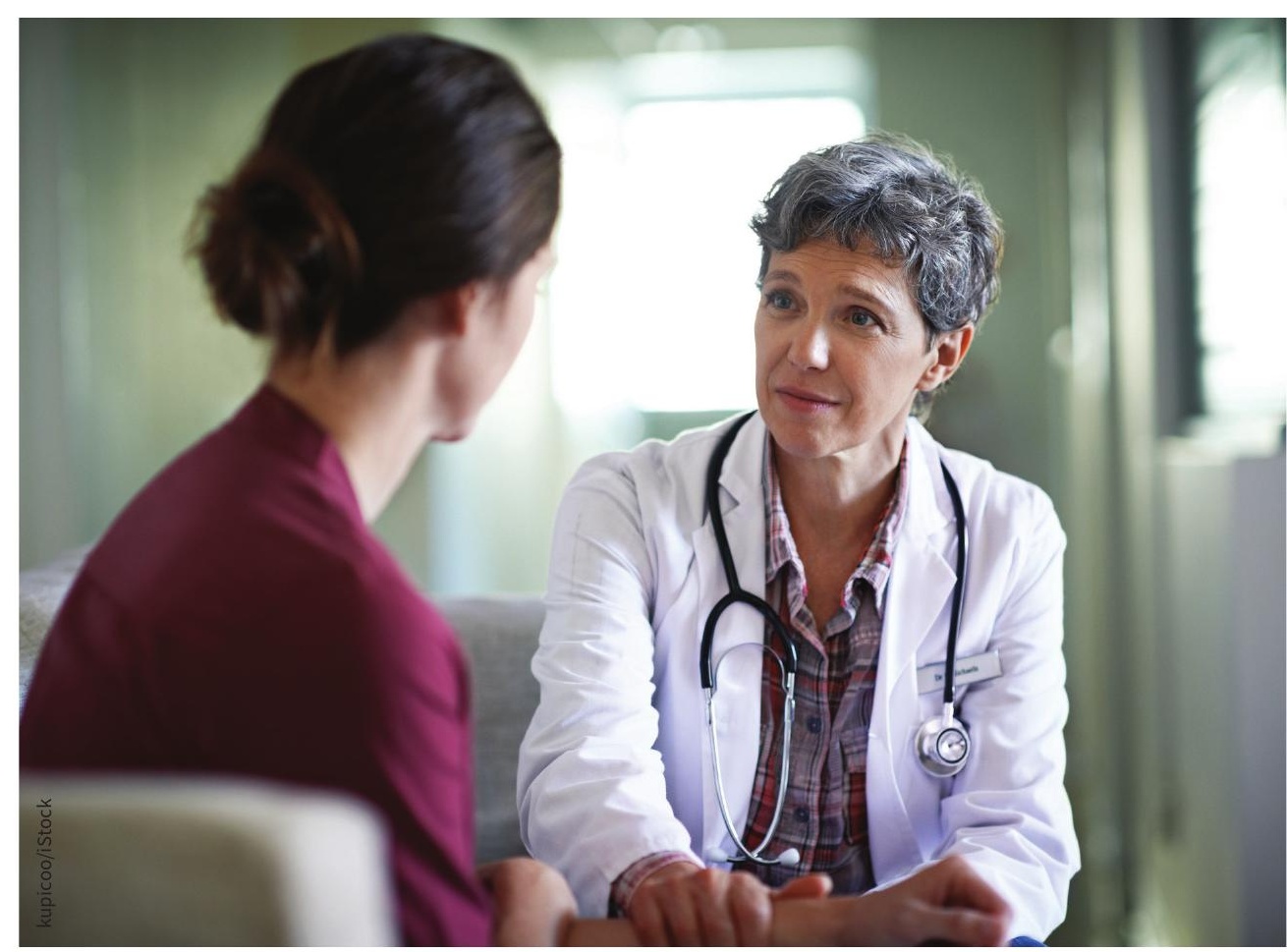

Access to medical abortion is improving but women still face hurdles.

from industry to the government, but it's not the standard of care that health professionals are held to," says Norman. "Clinical guidelines show that ultrasound is not a requirement."

The requirement is already causing concern in rural and remote areas, says Frédérique Chabot, director of health pro- pitals and there are delays up to three weeks to access those services," she says.

According to Joyce Arthur, executive director of the Abortion Rights Coalition of Canada, even some abortion clinics in hospitals don't provide ultrasounds, "severely hampering their efforts to serve women." 
Norman notes that "doing blood work is much more accessible and timely for many patients." However, adverse events are rare in medical abortion and recent studies in the United States and Ireland show there's no higher risk of complications when women forgo an ultrasound or other tests.
The cost of Mifegymiso, about $\$ 400$, also remains a barrier to access in provinces that don't fully cover the drug. "In Saskatchewan, Manitoba, Prince Edward Island, Newfoundland and Labrador, people have to pay out of pocket if they're not insured or part of a government pro- gram," says Chabot. "We're hoping that the entire country will have universal access, but each part of this puzzle matters and we've had tremendous victories to address what was a bit of a false start."

Lauren Vogel, CMAJ 